\title{
Intuitionistic fuzzy recursive enumerable languages and its closure properties
}

\author{
M. Rajasekar ${ }^{1 *}$ and V. Sumathi ${ }^{2}$
}

\begin{abstract}
In this paper a new class of Fuzzy Turing Machine is introduced which is called Intuitionistic Fuzzy Turing Machine. This Intuitionistic Fuzzy Turing Machine uses $t$-norm for membership degree and its dual $t$-conorm for non-membership degree. This paper studies the relation between Intuitionistic Fuzzy Turing Machine and Nondeterministic Turing Machine and the Closure properties of family of Intuitionistic Fuzzy Recursive Enumerable Languages under Concatenation and Reverse of a language.
\end{abstract}

\section{Keywords}

Nondeterministic Intuitionistic Fuzzy Turing Machine, Intuitionistic Fuzzy Recursive Enumerable Language.

\section{AMS Subject Classification}

68Q45,03D110.

${ }^{1}$ Mathematics Section, Faculty of Engineering and Technology, Annamalai University, Annamalainagar-608002, India.

${ }^{2}$ Department of Mathematics, Annamalai University, Annamalainagar-608002, India.

*Corresponding author: ${ }^{1}$ mdivraj1962@gmail.com; ${ }^{2}$ vsumathigeerthana83@gmail.com

Article History: Received 24 March 2019; Accepted 09 May 2019

(C)2017 MJM

\section{Contents}

1 Introduction 351

2

3 352

4 Closure Properties of Intuitionistic Fuzzy Recursive Enumerable Languages ...................... 353

5

References.... .354 354

\section{Introduction}

Turing Machine is a simple mathematical model of a computer. Despite its simplicity, the Turing Machine models the computing capability of a general-purpose computer. The Turing Machine studied the class of languages its defines(called the Recursively Enumerable sets)[2].

The first attempts concerning formalization of the notion of Fuzzy algorithms of Turing Machines are dated in late 1960 when this notation was introduced by Zadeh. Recently, it has been observed that corresponding so-called classical Fuzzy Turing Machines.[6]

The Fuzzy set theory was proposed by Lotfi A.zadeh [7], in 1965, in order to generalize the discrete notions of mem- bership and non-membership of classical set theory to the interval $[0,1]$.

In [5] Antonio Diego Silva Farias et al and others studied several variations of Fuzzy Turing Machine using $t$-norm and their languages. Also they discussed Closure properties of Fuzzy Recursive Enumerable Languages under Fuzzy Concatenation, Fuzzy Intersection and Fuzzy Reverse.

In 1986 Atanassov [1] introduced the concept of an Intuitionistic Fuzzy set which is characterized by two functions expressing the degree of belongingness and the degree of non- belongingness, respectively, this idea, which is a natural generalization of standard Fuzzy set, seems to be useful in modeling many real life situations.

For a fixed set $X$, a Intuitionistic Fuzzy subset of $X$ is a function from $X$ to $[0,1]$ considered as the degree of membership and the degree of non-membership. An Intuitionistic Fuzzy language is a Intuitionistic Fuzzy subset of $\Sigma^{*}$.

The Intuitionistic Fuzzy Turing Machine is to consider two dual degrees for each transition, one as the degree of its membership and one as the degree of its non-membership. The duality of $*, *^{\prime}$ play an important role here [3]

This paper is organized as follows. Section 2 contains the definition of $t$-norm, $t$-conorm, Fuzzy language accepted by Fuzzy Turing Machine and Intuitionistic Fuzzy language accepted by Intuitionistic Fuzzy Turing Machine. In section 3 we discuss the Characterization of Intuitionistic Fuzzy Tur- 
ing Machine. In section 4, we discuss the Closure properties of family of Intuitionistic Fuzzy Recursive Enumerable Languages under Concatenation and Reverse of a language.

\section{Preliminaries}

Definition 2.1. [5] At-norm is a function $T:[0,1] \times[0,1] \rightarrow$ $[0,1]$ which satisfies the following properties:

(T1) $T(x, 1)=x$, for any $x \in[0,1]$;

(T2) $T(x, y)=T(y, x)$, for all $x, y \in[0,1]$;

(T3) $T(x, y) \leq T(x, z)$, for any $x, y, z \in[0,1]$ with $y \leq z$;

(T4) $T(x, T(y, z))=T(T(x, y), z)$ for any $x, y, z \in[0,1]$.

Definition 2.2. [5] A $t$-conorm is a function $S:[0,1] \times$ $[0,1] \rightarrow[0,1]$ which satisfies the following properties:

(S1) $S(x, 0)=x$, for any $x \in[0,1]$;

(S2) $S(x, y)=S(y, x)$, for all $x, y \in[0,1]$;

(S3) $S(x, y) \leq S(x, z)$, for any $x, y, z \in[0,1]$ with $y \leq z$;

(S4) $S(x, S(y, z))=S(S(x, y), z)$ for any $x, y, z \in[0,1]$.

Definition 2.3. [6] The fuzzy language accepted by $\mathscr{F}$ is the fuzzy set of ordered pairs $L(\mathscr{F})=\{(w, e(w)) \mid w$ is accepted by $\mathscr{F}$ with truth degree $e(w)\}$.

Definition 2.4. [4] A Nondeterministic Intuitionistic Fuzzy Turing Machine NIFTM is defined by

$\left(Q, \sum, \Gamma, \Delta, q_{0}, \square, F, *, *^{\prime}, \mu, \gamma\right)$ where

$Q$ is a finite set of states,

$\sum$ is the input alphabets,

$\Gamma$ is a finite set of symbols called the tape alphabets,

$\Delta$ is an intuitionistic fuzzy subsets of

$Q \times \Gamma \times 2^{Q \times \Gamma \times\{L, R\}}$ and

$\mu, \gamma: Q \times \Gamma \times 2^{Q \times \Gamma \times\{L, R\}} \rightarrow[0,1]$ are

functions where $\mu(\delta)+\gamma(\delta) \leq 1 \quad \forall \delta \in \Delta$,

$q_{0} \in Q$ is the initial state,

$\square \in \Gamma$ is a special symbol called the blank,

$F \subseteq Q$ is the set of accepting states,

$*$ is a computable $t-n o r m$ and $*^{\prime}$ is its computable $t-$ conorm.

\section{Definition 2.5. [4]}

An Intuitionistic Fuzzy language accepted by a Nondeterministic Intuitionistic Fuzzy Turing Machine is defined by

$$
\begin{aligned}
& L(M)=\left\{(w,(\mu, \gamma)) \mid w \in \Sigma^{+},\left(q_{0} w,(1,0)\right) \stackrel{\vdash}{\vdash}^{*}\right. \\
& \left.\left(u^{\prime} q_{f} v^{\prime},\left(\mu_{t}, \gamma_{t}\right)\right), q_{f} \in F, u^{\prime}, v^{\prime} \in \Gamma^{*}\right\} .
\end{aligned}
$$

\section{Characterization of Intuitionistic Fuzzy Turing Machines}

Theorem 3.1. Let $\mathscr{M}=\left(Q, \sum, \Gamma, \Delta, q_{0}, \square, F, *, *^{\prime}, \mu, \gamma\right)$ be an intutionistic Fuzzy Turing Machine that accepts Intuitionistic Fuzzy language $L(\mathscr{M})$. Then there exist a nondeterministic Turing Machine $M$ that accept crisp language $L_{1}$ of $L(\mathscr{M})$ that is $\operatorname{crisp}(L(\mathscr{M}))=L_{1}(M)$
Proof. We show that for any classical Intuitionistic Fuzzy Turing Machine $\mathscr{M}=\left(Q, \sum, \Gamma, \Delta, q_{0}, \square, F, *, *^{\prime}, \mu, \gamma\right)$ with a computable $t-$ norm and $t$-conorm there exist a recursive enumerable language $L_{1}$ accepted by nondeterministic Turing Machine $M$ such that $(w,(\mu, \gamma)) \in L(\mathscr{M})$ if and only if $w \#(\mu, \gamma) \in L_{1}(M)$ for any $w \in\left(\sum-\{\#\}\right)^{*}$ and $\# \in \Gamma$. Let $M$ be a "defuzzified" crisp version of $\mathscr{M}$ that is in nondeterministic Turing Machine $M$ all instructions have membership degree 1 and non-membership degree 0 . Let $A D_{\mathscr{M}}(w)$ denote the set of all acceptance paths that lead from initial instantaneous descriptions of $Q_{0}$ to accepting instantaneous descriptions $Q_{t}$, let $\left(e_{1}, e_{2}\right)$ be the evaluation function that to each $I D$ assigning its membership degree and non-membership degree. Consider the commutative ordered semiring $R=\left([0,1], *, *^{\prime}, \leq\right)$ where $*$ is a $t-$ norm and $*^{\prime}$ is a $t$-conorm.

Let $I=\left\{\alpha_{1}<\alpha_{2}<\ldots<\alpha_{m}\right\} \subset[0,1] \subset Q$ be the set of instruction membership degrees and non-membership degrees, that is the range of $\mu$ and $\gamma$. Let $R(I)$ be a subsemiring of $R$ generated by $I$. Syntactically, the elements of $R(I)$ are formed by products (via operator $*$ and $*$ ' depending the $t$-norm and $t$-conorm) of elements of $I$ that can be seen as tuples with entries from $I$. Define language $L_{1}=\{w \#(\mu, \gamma) \mid \exists \delta \in$ $A D_{\mathscr{M}}(w): e_{1}(\delta)=\mu, e_{2}(\delta)=\gamma$ and $\left.0 \leq \mu+\gamma \leq 1\right\}$. Then we will show that $L_{1}$ is a recursive enumerable languages accepted by $M$. We claim that the nondeterministic Turing machine $M$ that on input $w \#(\mu, \gamma)$ guesses the tuple representation of $(\mu, \gamma)$, calculates its numerical value and verifies whether $\mu$ and $\gamma \in R(I)$ such that $0 \leq \mu+\gamma \leq 1$. Then $M$ guesses $\delta=(\mu, \gamma)$ and simulates $\mathscr{M}$ on $w$ to see whether $\delta \in A D_{\mathscr{M}}(w)$ and whether $e_{1}(\delta)=\mu$ and $e_{2}(\delta)=\gamma$. Hence $\left(w,(\mu, \gamma) \in L(M)\right.$ if and only if $w \#(\mu, \gamma) \in L_{1}(M)$.

Theorem 3.2. Let $M=\left(Q, \sum, \Gamma, \Delta, q_{0}, \square, F\right)$ be a nondeterministic Turing Machine that accepts recursive enumerable Language $L_{1}$. Then there exists an Intuitionistic Fuzzy Turing Machine that accepts the language $L_{1}$.

Proof. Let $L_{1}$ be a recursive enumerable Language. There is a nondeterministic Turing $M$ recognizing $L_{1}$. We will show that there is a classical Intutionistic Fuzzy Turing Machine $\mathscr{M}=\left(Q, \sum, \Gamma, \Delta, q_{0}, \square, F, *, *^{\prime}, \mu, \gamma\right)$ such that $w \in L_{1}$ if and only if $w$ is accepted by $\mathscr{M}$ with membership degree 1 and non-membership degree 0 (that is $(w,(1,0)) \in L(\mathscr{M})$ ). In $\mathscr{M}$, unless otherwise stated, all instructions will have membership degree 1 and non-membership degree 0 . On input $w$ of $\mathscr{M}$ takes a nondeterministic branch. When accepting path of the Turing Machine $M$ is reached $\mathscr{M}$ enters an accepting state $q_{f}$ with membership degree 1 and non-membership degree 0 .

That is $\left(q_{0} w,(1,0)\right) \stackrel{*}{\vdash} \mathscr{M} \quad\left(u q_{f} v,(\mu, \gamma)\right)$ where

$$
\begin{aligned}
& \mu=*^{\prime}(1 * 1 * \ldots * 1) \\
& \mu=1 \\
& \gamma=*\left(0 *^{\prime} 0 *^{\prime} \ldots *^{\prime} 0\right) \\
& \gamma=0
\end{aligned}
$$

Therefore $(w,(1,0)) \in L(\mathscr{M})$. 


\section{Closure Properties of Intuitionistic Fuzzy Recursive Enumerable Languages}

Definition 4.1. Let $L_{1}$ and $L_{2}$ be an Intuitionistic Fuzzy languages accepted by Machine

$M=\left(Q, \sum, \Gamma, \Delta, q_{0}, \square, F, *, *^{\prime}, \mu, \gamma\right)$ then concatenation of $L_{1} L_{2}$ is defined by

$L_{1} L_{2}=\left\{(u v,(\mu, \gamma)) \mid u \in L_{1}, v \in L_{2}, \mu=*_{u}^{\prime}\left(\mu_{L_{1}}(u) * \mu_{L_{2}}(v)\right), \gamma=\right.$ $\left.*\left(\gamma_{L_{1}}(u) *^{\prime} \gamma_{L_{2}}(v)\right)\right\}$

Theorem 4.2. Let $L_{1}$ and $L_{2}$ be two Intuitionistic Fuzzy languages on the same input alphabet. If there are NIFTM's $M_{1}=\left(Q_{1}, \sum, \Gamma, \Delta_{1}, q_{0}^{1}, \square, F_{1}, *_{1}, *_{1}^{\prime}, \mu_{1}, \gamma_{1}\right)$,

$M_{2}=\left(Q_{2}, \Sigma^{\prime}, \Gamma^{\prime}, \Delta_{2}, q_{0}^{2}, \square, F_{2}, *_{2}, *_{2}^{\prime}, \mu_{2}, \gamma_{2}\right)$ with $L\left(M_{1}\right)=L_{1}$ and $L\left(M_{2}\right)=L_{2}$. Then there is a NIFTM $M$ such that $L(M)=$ $L_{1} L_{2}$.

Proof. Given two NIFTM's

$M_{1}=\left(Q_{1}, \sum, \Gamma, \Delta_{1}, q_{0}^{1}, \square, F_{1}, *_{1}, *_{1}^{\prime}, \mu_{1}, \gamma_{1}\right)$,

$M_{2}=\left(Q_{2}, \Sigma^{\prime}, \Gamma^{\prime}, \Delta_{2}, q_{0}^{2}, \square, F_{2}, *_{2}, *_{2}^{\prime}, \mu_{2}, \gamma_{2}\right)$ on the same input alphabet, where $\Gamma \subseteq \Sigma^{\prime}$, we build a new NIFTM, denoted by $M=\left(Q, \sum, \Gamma, \Delta, q_{0}^{\prime}, \square, F, *, *^{\prime}, \mu, \gamma\right)$ where (i) we can assume without loss of generality that, we can assume that $Q_{1} \cap Q_{2}=\phi$ and define $Q=Q_{1} \cup Q_{2}$;

(ii) $q_{0}=q_{0}^{\prime}$;

(iii) $F=F_{2}$;

(iv) $\Delta=\Delta_{1} \cup \Delta_{2} \cup\left\{\left(\ell=(1,0)=\left(q, a, q_{0}^{2}, a, L\right)\right.\right.$ for some $q \in$ $F_{1}$ where $\left.a \in \Gamma^{\prime}\right\}$ and for $\delta=(\mu, \gamma), \delta^{\prime}=\left(\mu^{\prime}, \gamma^{\prime}\right) \in \Delta$

$$
\begin{aligned}
& \mu(\delta)=\left\{\begin{array}{l}
1 \text { if } \mu(\ell)=1 ; \\
\mu_{1}(\delta) \text { if } \delta \in \Delta_{1} ; \\
\mu_{2}(\delta) \text { if } \delta \in \Delta_{2} .
\end{array}\right. \\
& \gamma(\delta)=\left\{\begin{array}{l}
0 \text { if } \gamma(\ell)=0 ; \\
\gamma_{1}(\delta) \text { if } \delta \in \Delta_{1} ; \\
\gamma_{2}(\delta) \text { if } \delta \in \Delta_{2} .
\end{array}\right. \\
& \mu * \mu^{\prime}=\left\{\begin{array}{l}
\mu *_{1} \mu^{\prime} \text { if } \delta, \delta^{\prime} \in \Delta_{1} ; \\
\mu *_{2} \mu^{\prime} \text { if } \delta, \delta^{\prime} \in \Delta_{2} .
\end{array}\right. \\
& \gamma *^{\prime} \gamma^{\prime}=\left\{\begin{array}{l}
\gamma *_{1}^{\prime} \gamma^{\prime} \text { if } \delta, \delta^{\prime} \in \Delta_{1} ; \\
\gamma *_{2}^{\prime} \gamma^{\prime} \text { if } \delta, \delta^{\prime} \in \Delta_{2} .
\end{array}\right.
\end{aligned}
$$

Let $(w=u v,(\mu, \gamma)) \in L(M)$

$$
\begin{aligned}
& \left(q_{0}^{\prime} u v,(1,0)\right){ }^{\prime} M_{1}\left(u^{\prime} q_{f_{1}} v^{\prime},\left(\mu_{t_{1}}, \gamma_{t_{1}}\right) \vdash^{*}\left(q_{0}^{2} u^{\prime} v^{\prime},(1,0)\right)\right. \\
& \vdash_{M_{2}}\left(u^{\prime \prime} q_{f_{2}} v^{\prime \prime},\left(\mu_{t_{2}}^{\prime}, \gamma_{t_{2}}^{\prime}\right)\right) \\
& \text { where } \mu=*^{\prime}\left(\left(\mu_{1} * \mu_{2} * \ldots * \mu_{t_{1}}\right) * 1\right. \\
& \left.\quad *\left(\mu_{1}^{\prime} * \mu_{2}^{\prime} * \ldots * \mu_{t_{2}}^{\prime}\right)\right) \\
& \mu=*^{\prime}\left(\left(\mu_{1} *{ }_{1} \mu_{2} *_{1} \ldots * *_{1} \mu_{t_{1}}\right) * 1\right. \\
& \left.*\left(\mu_{1}^{\prime} * 1 \mu_{2}^{\prime} *_{1} \ldots * 1 \mu_{t_{2}}^{\prime}\right)\right) \\
& \mu=*^{\prime}\left(\left(\mu_{1} *_{1} \mu_{2} *_{1} \ldots * *_{1} \mu_{t_{1}}\right)\right.
\end{aligned}
$$

$$
\begin{aligned}
& \left.*\left(\mu_{1}^{\prime} *_{1} \mu_{2}^{\prime} *_{1} \ldots *_{1} \mu_{t_{2}}^{\prime}\right)\right) \\
& \mu=*_{u}^{\prime}\left(r_{1} * r_{1}^{\prime}\right) \\
& \mu=*_{u}^{\prime}\left(\mu_{L_{1}}(u) * \mu_{L_{2}}(v)\right) \\
& \gamma=*\left(\left(\gamma_{1} *^{\prime} \gamma_{2} *^{\prime} \ldots *^{\prime} \gamma_{t_{1}}\right) *^{\prime} 0\right. \\
& \left.*^{\prime}\left(\gamma_{1}^{\prime} *^{\prime} \gamma_{2}^{\prime} *^{\prime} \ldots *^{\prime} \gamma_{t_{2}}^{\prime}\right)\right) \\
& \gamma=*\left(\left(\gamma_{1} *_{1}^{\prime} \gamma_{2} *_{1}^{\prime} \ldots *_{1}^{\prime} \gamma_{t_{1}}\right)\right. \\
& \left.*^{\prime} 0 *^{\prime}\left(\gamma_{1}^{\prime} *_{2}^{\prime} \gamma_{2}^{\prime} *_{2}^{\prime} \ldots *_{2}^{\prime} \gamma_{t_{2}}^{\prime}\right)\right) \\
& \gamma=*\left(\left(\gamma_{1} *_{1}^{\prime} \gamma_{2} *_{1}^{\prime} \ldots *_{1}^{\prime} \gamma_{t_{1}}\right) *^{\prime}\left(\gamma_{1}^{\prime} *_{2}^{\prime} \gamma_{2}^{\prime} *_{2}^{\prime} \ldots *_{2}^{\prime} \gamma_{t_{2}}^{\prime}\right)\right) \\
& \gamma=*\left(r_{2} *^{\prime} r_{2}^{\prime}\right) \\
& \gamma=*\left(\gamma_{L_{1}}(u) *^{\prime} \gamma_{L_{2}}(v)\right) \\
& \text { Therefore }(u v,(\mu, \gamma)) \in L_{1} L_{2} \text {, } \\
& \text { conversely }(u v,(\mu, \gamma)) \in L_{1} L_{2} \\
& \mu=*_{u}^{\prime}\left(\mu_{L_{1}}(u) * \mu_{L_{2}}(v)\right) \\
& \Rightarrow \mu=*_{u}^{\prime}\left(r_{1} * r_{1}^{\prime}\right) \\
& \mu=*_{u}^{\prime}\left(r_{1} * 1 * r_{1}^{\prime}\right) \\
& \mu=*_{u}^{\prime}\left(r_{1} * r_{1}^{\prime}\right) \\
& \gamma=*\left(\gamma_{L_{1}}(u) *^{\prime} \gamma_{L_{2}}(v)\right) \\
& \gamma=*\left(r_{2} *^{\prime} r_{2}^{\prime}\right) \\
& \gamma=*\left(r_{2} *^{\prime} 0 *^{\prime} r_{2}^{\prime}\right) \\
& \gamma=*\left(r_{2} *^{\prime} r_{2}^{\prime}\right)
\end{aligned}
$$

Theorem 4.3. Let $L$ be an Intuitionistic Fuzzy language. If there is a nondeterministic Intuitionistic Fuzzy Turing Machine $\mathscr{M}=\left(Q, \sum, \Gamma, \Delta, q_{0}, \square, F, *, *^{\prime}, \mu, \gamma\right)$ such that $L(\mathscr{M})=$ $L$ then there is a nondeterministic Intuitionistic Fuzzy Turing Machine $\mathscr{M}^{\prime}$ such that $L\left(\mathscr{M}^{\prime}\right)=L^{R}$

Proof. $\quad \operatorname{In}[2]$ find a nondeterministic Turing Machine $M_{1}$ which converts string $w \in \sum^{*}$ into reverse $w^{R}$ that is $q_{0} w \stackrel{*}{\vdash}_{M}$ $q_{f}^{\prime} w^{R}$. By the theorem 3.2 there exists a nondeterministic Intuitionistic Fuzzy Turing Machine $\mathscr{M}_{1}$ such that $\left(q_{0} w,(1,0)\right) \vdash^{*} \mathscr{M}_{1}$ $\left(q_{f}^{\prime} w^{R},(1,0)\right)$. We concatenate the nondeterministic Intuitionistic Fuzzy Turing Machine $\mathscr{M}_{1}$ with $\mathscr{M}$ we obtain another nondeterministic Intutionistic Fuzzy Turing Machine $\mathscr{M}^{\prime}=\mathscr{M}_{1} \mathscr{M}$. Now we show that this new nondeterministic Intuitionistic Fuzzy Turing Machine is accept $L\left(\mathscr{M}^{\prime}\right)=L^{R}$, since for all $w \in \Sigma^{*}$ with $\left(q_{0} w,(1,0)\right) \stackrel{*}{\vdash} \mathscr{M}\left(u q_{f} v,(\mu, \gamma)\right)$, accepted by $\mathscr{M}$, we have $\left(q_{0} w^{R},(1,0)\right) \stackrel{*}{\vdash^{\prime}} \mathscr{M}_{1}\left(q_{f}^{\prime} w,(1,0)\right) \stackrel{*}{\vdash} \mathscr{M}$ $\left(q_{0} w,(1,0)\right) \stackrel{*}{\vdash} \mathscr{M}\left(u q_{f} v,(\mu, \gamma)\right)$.

This shows that $\left(q_{0} w,(1,0)\right) \vdash_{\mathscr{M}}\left(u q_{f} v,(\mu, \gamma)\right)$ $\Rightarrow\left(q_{0}^{\prime} w^{R},(1,0)\right) \vdash^{*} \mathscr{M}^{\prime}\left(u q_{f} v,(\mu, \gamma)\right)$

Therefore nondeterministic Intuitionistic Fuzzy Turing Machine $\mathscr{M}^{\prime}$ accept the language $L^{R}$. 


\section{Conclusion}

The results presented in this paper are part of a continuing research on the foundations of Intuitionistic Fuzzy Recursive Enumerable languages. This paper introduces a new, narrower sense of the concept of an Intuitionistic Fuzzy Recursive Enumerable Languages. Usually Intuitionistic Fuzzy Turing Machine comes equipped with a connective operator, a $t-n o r m$ and $t$-conorm, so we can compute the degree of acceptance and non acceptance of a string and thus determine the Intuitionistic Fuzzy language accepted by an Intuitionistic Fuzzy Turing Machine. This paper discuss the relation between Intuitionistic Fuzzy Turing Machine and Nondeterministic Turing Machine and also establish the Closure properties of family of Intuitionistic Fuzzy Recursive Enumerable Languages under Concatenation and Reverse of a language.

\section{Acknowledgment}

The work of the second author is supported by the "University Research Fellowship".

\section{References}

[1] Atanassov. K, Intuitionistic Fuzzy sets, Fuzzy Sets and Systems, vol.20, 1986, pp 87-96.

[2] J. E. Hopcroft and J. D. Ullman, Introduction to Automata Theory, Lanuanges and Computation, New York: Addison-Wesley, 1979.

[3] M. Moniri, Fuzzy and Intuitionistic Fuzzy Turing Machines, Fundamenta Informaticae, vol.123, 2013, pp 305315.

[4] M. Rajasekar and V. Sumathi, Intuitionistic Fuzzy Recursive Enumerable Languages, journal of JETIR, vol.5, no. 10, 2018

[5] Antonio Diego Silva Farias, Luiz Ranyer de Araujo Lopes, Benjamin Bedregal and Regivan H.N. Santiago., Closure properties for fuzzy recursively enumerable languaes and fuzzy recursive languages, Journal of Intelligent and Fuzzy systems, vol.31, 2016, pp 1795-1806.

[6] J. Wiedermann, Characterizing the super-Turing computing power and efficiency of classical fuzzy Turing machines, Theoretical Computer science, vol.317, 2004, pp 61-69.

[7] L. A. Zadeh, Fuzzy sets, Inform control, vol. 8, 1965, pp 338-353.

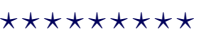

ISSN(P):2319-3786

Malaya Journal of Matematik

$\operatorname{ISSN}(\mathrm{O}): 2321-5666$

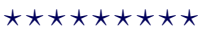

